\title{
Kernos
}

Revue internationale et pluridisciplinaire de religion grecque antique

13 | 2000

Varia

\section{Juan Antonio LóPEZ FÉREZ (éd.), La comedia griega y su influencia en la literatura española}

\section{Angel Ruiz Pérez}

\section{OpenEdition \\ Journals}

\section{Édition électronique}

URL : http://journals.openedition.org/kernos/1348

DOI : $10.4000 /$ kernos. 1348

ISSN : 2034-7871

\section{Éditeur}

Centre international d'étude de la religion grecque antique

Édition imprimée

Date de publication : 1 janvier 2000

ISSN : 0776-3824

\section{Référence électronique}

Angel Ruiz Pérez, « Juan Antonio LóPEz férez (éd.), La comedia griega y su influencia en la literatura española », Kernos [En ligne], 13 | 2000, mis en ligne le 21 avril 2011, consulté le 24 septembre 2020. URL : http://journals.openedition.org/kernos/1348; DOI : https://doi.org/10.4000/kernos.1348 
le salut mystique, la fusion et l'identification avec la divinité. Cette ascèse peut s'opérer par la mortification des impulsions de la libido (in ipo) ou au contraire en les exaltant à leur paroxysme (in iper). Dans ce chapitre, l'auteur passe en revue les diverses réponses qui ont été apportées au problème de la destinée individuelle après la mort. Parmi les réponses non gnostiques, il retient celles fondées sur l'idée d'une résurrection, qui constituèrent les antécédents nécessaires sur lesquels s'exerça la réflexion de personnages qui ont ouvert la voie au gnosticisme, la réponse d'un juif alexandrin, Philon, et celle d'un juif palestinien, Jésus. Parmi les réponses gnostiques, il retient celle de Ménandre, défenseur de ce que les écrivains orthodoxes de la fin du $\mathrm{I}^{\mathrm{er}}$ siècle et du début $\mathrm{du} \mathrm{II}^{\mathrm{e}}$ siècle appelèrent " la fausse gnose », celle de Cérinte, pour terminer avec les deux voies des gnostiques de Barbelo et de Seth, la voie de gauche et la voie de droite. La première, dans une ascèse "proprement libertine » vise à atteindre la perfection par le coït et à combattre le plaisir par le plaisir; la seconde, au contraire, réprime l'instinct sexuel et prône une technique de méditation. Ce deuxième chapitre marque la fin de l'ouvrage de Casadio et n'est suivi que de la table des matières. Si le contenu des deux chapitres ne manque pas d'intérêt, on peut regretter que le programme annoncé dans l'introduction n'ait pas été réalisé : le lecteur, qui se sera par ailleurs passé de conclusion, de bibliographie et d'index, aura assuré luimême le lien entre les deux parties de l'ouvrage.

Carine Van Liefferinge (Université libre de Bruxelles)

Juan Antonio López Férez (éd.), La comedia griega y su influencia en la literatura española, Madrid, Ediciones Clásicas, 1999. 1 vol. $15 \times 21 \mathrm{~cm}$, vIII+490 p. (Estudios de Filologia Griega, 3). ISBN : 84-7882-343-3.

Le professeur López Férez, de l'Universidad Nacional de Educación a Distancia, continue à organiser à Madrid des congrès sur des questions littéraires centrales; jusqu'à maintenant, il a publié trois volumes d'actes sur des thèmes concrets, comprenant une mise à jour sur un genre littéraire, avec des études complémentaires à propos de l'influence du genre dans la littérature occidentale, spécialement espagnole. Les auteurs sont des professeurs bien connus pour leurs études sur la comédie grecque, tant en Espagne que dans d'autres pays européens. Le livre comporte un index de textes cités (p. 457-471), de noms propres et de thèmes particuliers (p. 472-488) et d'une liste des auteurs du volume (p. 489490).

Francisco Rodríguez Adrados ("Comedia y tragedia", p. 1-22) fait le prologue du livre qui contient des réflexions générales sur les points communs entre la tragédie et la comédie, et sur leur influence dans le théâtre occidental. On doit mettre à part le travail de Luis Gil Fernández ("La risa y lo cómico en el pensamiento antiguo", p. 307-343), puisqu'il fait une étude générale sur la théorie littéraire grecque et latine dans le domaine de la comédie. Le professeur Gil étudie tout d'abord le rire en tant que phénomène physiologique et social, mais le noud de son exposé est consacré à la théorie littéraire sur le rire, le comique et la comédie, spécialement chez Platon, Aristote, Cicéron, Quintilien et le théoricien prébyzantin Platonius. - La plupart des contributions tournent autour de la comédie archaïque. Giuseppe Mastromarco ("La commedia antica fra tradizione e innovazione", p. 23-42, à partir de son travail sur la comédie dans Lo spazio letterario della Grecia antica, I, 1) fait une analyse générale et préfère ne pas distinguer trop précisément les deux courants : celui des auteurs politiques ou « engagés » (Cratinos, Magnète) et celui des auteurs de fiction; on peut remarquer que chez Aristophane sont présents des traits des deux tendances : ce qui vient de 
la farce dorienne, les thèmes traditionnels se mêlent à la thématique «politique ", mais après l'année 404, on y trouve toujours les thèmes de la tradition. Dans le même esprit, l'article d'Antonio Melero ("Los predecesores de Aristófanes", p. 6380) veut approfondir l'étude des petits fragments et témoignages sur d'《autres » auteurs de la comédie archaïque, suivant un schéma que l'on pourrait qualifier de "généalogique ». - Alan H. Sommerstein ("The Theatre Audience and the Demos", p. 43-62) envisage la comédie archaïque du point de vue du public d'Aristophane; il signale le danger d'identifier l'audience avec l'ensemble des citoyens d'Athènes; n'entraient que ceux qui pouvaient payer le prix de la place (première limitation), c'est-à-dire les citoyens des couches sociales élevées et les métèques, aux idées traditionnelles : cela obligerait les auteurs à acquérir "a rightwing persona"; ce n'était pas le cas d'Aristophane, dont les idées n'entraient pas en contradiction avec les exigences du public, sauf dans le cas de sa faveur pour Sparte. - Deux contributions analysent la forme scénique. La première est celle de Pascal Thiercy ("L'évolution de l'action dans les comédies d'Aristophane", p. 81 100) qui considère la problématique de l'unité d'action et d'intérêt chez Aristophane, question complexe à laquelle il répond de manière affirmative, sauf pour Les femmes à l'Assemblée. La deuxième contribution est celle d'Ignacio Rodríguez Alfageme ("La forma escénica de la Comedia antigua. Un ejemplo: los Acarnienses", p. 113-135) qui part du concept de "scène " (pour chaque fragment les mêmes personnages restent en scène) et organise le texte des Acharnaniens selon une structure étonnamment régulière, avec quatre séquences de treize scènes, en plus de l'exode. Il a continué cette recherche dans le reste de la production d'Aristophane (cf. Cuadernos de Filología Clásica. Estudios Griegos e Indoeuropeos, 21 (1998) avec des réferences). - Antonio López Eire fait dans son article ("Sobre el ático coloquial de la Comedia aristofánica", 137-175) une étude linguistique mais aussi une belle leçon de traduction du grec "parlé », qu'on lit d'autant plus agréablement qu'il adopte un ton humoristique ici et là. - Bernhard Zimmermann analyse ("Aristofane e la crisi dell'educazione ateniese", p. 101-112) le personnage type de l'intellectuel, surtout à partir des Nuées et des Oiseaux. Ce personnage est caractérisé comme le savant distrait de l'anecdote de Thalès, mais aussi comme membre d'une caste fermée et arrogante. - Pour sa part Emilio Suárez de la Torre ("Observaciones sobre la presencia. de la mántica en la Comedia griega", p. 177-201) propose d'étudier la présence de la mantique dans les cuvres d'Aristophane par rapport à la tradition dans laquelle la comédie archaïque s'insère, celle de la poésie parodique-satirique archaïque, mais aussi par rapport à ses propres comédies, en réorganisant cès éléments dans un argument concret pour servir aux besoins de la représentation dramatique; on doit ajouter aussi le contexte historique et l'influence du public. Grâce à ces rapprochements, on peut mieux comprendre l'évolution de la chresmologie et la valeur des oracles dans l'œuvre d'Aristophane. - Avec Franca Perusino ("Tra commedia antica e commedia nuova: considerazione sul ruolo della commedia di mezzo nella cultura greca del IV secolo", p. 203-214) on laisse l'étude de la comédie archaïque pour tenter de délimiter la comédie moyenne, malgré le manque de témoins sûrs. Platonius, bien connu par Perusino, apporte deux nouveautés : l'abolition des chants du chœur (que l'on doit interpréter au sens où le chœur n'intervient plus dans l'action) et de la parabase, et aussi de l'attaque personnelle et directe, remplacée par la parodie des récits mythiques, en prenant comme modèle l'Eolosicon d'Áristophane, de 387. La perte de fonction du chour est déterminante : le public n'est pas représenté dans l'action, et c'est pourquoi la composante politique s'affaiblit nécessairement, ouvrant la voie à la comédie nouvelle, qui ne comprend pas non plus la parodie du mythe. - Enzo Degani ("L'elemento gastronomico nella commedia greca postaristofanea", p. 215-225) 
trouve des antécédents de l'utilisation parodique de la gastronomie dans la poésie iambique et le drame satirique, mais Épicarme deviendra celui qui introduira des personnages gourmands, ivres ou cuisiniers. Dans toute la comédie athénienne, la nourriture est un motif humoristique, mais spécialement dans le "filone disimpegnato » de la comédie archaïque et dans la comédie postérieure. - Carmen Morenilla Talens ("El hetaîros en Menandro", p. 227-269) étudie le couple formé par le protagoniste et son ami qui l'aide, ce dernier parfois remplacé dans ses fonctions par un parasite ou un serviteur, dans un schéma dramatique qui aura un grand succès dans la suite. - Le travail du professeur Jesús Lens Tuero, récemment décédé, auquel le prof. López Férez dédie sa contribution, ("Comedia e Historiografía: Ctesias de Cnido", p. 271-306) porte sur les concommitances entre les procédés historiographiques de Ctésias de Cnide et ceux de la comédie, au sujet des endroits, des personnages, des structures narratives, des types fixés et quelque peu stéréotypés. - Pour conclure, il y a trois articles sur l'influence de ce genre dans la littérature postérieure: Andrés Pociña ("Menandro en la comedia latina", p. 345-367) observe l'influence de cet auteur à Rome; Antonio Bravo García ("El Aristófanes de las bibliotecas de la Comunidad de Madrid: una ojeada a los fondos de El Escorial", 369-386) fait une révision des fonds de l'Escorial en ce qui concerne Aristophane, du point de vue codicologique et paléographique, et l'éditeur du volume, Juan Antonio López Férez fait un "Estudio sobre la influencia de la comedia griega en la literatura española", (387455), nécessairement rapide mais avec une grande quantité de données, une source importante pour des études plus détaillées.

Angel Ruiz Pérez
(Universidad de Santiago de Compostela)

Philippe Borgeaud (avec Nicole Durisch, Antje Kolde et Grégoire Sommer), La mythologie du matriarcat. L'atelier de Johann Jakob Bachofen, Genève, Droz, 1999. 1 vol. $15 \times 22 \mathrm{~cm}, 252$ p. (Recherches et Rencontres. Publications de la Faculté des lettres de Genève, 13). ISBN : 2-600-00350-9.

Le mot de «matriarcat » fait immédiatement surgir tout un univers fantasmatique où les femmes ont le pouvoir et l'exercent. Certains mythes anciens et certaines enquêtes ethnologiques, qu'elles soient antiques ou du temps des Lumières, évoquent en effet une série de peuplades ayant connu une domination féminine, en plus de la simple filiation matrilinéaire. Ces récits ont profondément marqué Johann Jakob Bachofen, qui a publié sur le sujet, en 1861, un gros ouvrage intitulé Das Mutterrecht, Le Droit maternel (désormais disponible dans la traduction française d'Étienne Barilier, Lausanne, 1996). Ph. Borgeaud s'est penché, avec quelques collaborateurs, sur les archives de Bachofen conservées à Bâle, pour éclairer le cheminement souvent tortueux et la longue maturation qui l'ont conduit à élaborer ses théories et à devenir ainsi le fondateur de l'idée matriarcale, même si le mot comme tel ne se trouve pas dans l'œuvre. L'étude de $\mathrm{Ph}$. Borgeaud nous ouvre donc bien la porte de "l'atelier de J.J. Bachofen ». L'ouvrage fait tout d'abord le point sur la notion de " gynécocratie ». L'analyse du mot et de la chose en Grèce ancienne montre qu'il s'agit essentiellement d'une exploration imaginaire des limites du social, qui inverse les valeurs fondatrices de la communauté citoyenne. Mais c'est dans la perspective d'une véritable genèse historique, et pas seulement d'un renversement symbolique des valeurs, que Bachofen, après Lafitau mais sans lien de dépendance avec ce dernier, va progressivement penser le «droit maternel ». Par contre, la dette de Bachofen à l'égard des théories de son temps sur les notions de mythe et de symbole est assurée : Heyne, Creuzer, Gerhard, K.O. Müller, Preller forment l'horizon intellectuel sur 